\title{
Strain-Enhanced Ionic Conductivity
}

\author{
T.J. Pennycook, ${ }^{* * *}$ M. Varela, ${ }^{* *}$ M.J. Beck, ${ }^{*}$ J. Garcia-Barriocanal,*** F. Y. Bruno, ${ }^{* * *}$ C. Leon, ${ }^{* * *}$ J. \\ Santamaria, ${ }^{* *}$ S.T. Pantelides, ${ }^{*}, *$ and S.J. Pennycook**** \\ * Department of Physics and Astronomy, Vanderbilt University, Nashville, TN 37235 \\ ** Materials Science and Technology Division, Oak Ridge National Laboratory, PO Box 2008, Oak \\ Ridge, TN 37831-6030 \\ *** Complutense University, 28040 Madrid, Spain
}

High ionic conductivities are essential for fuel cells to operate efficiently. Solid-oxide fuel cells are one of the most efficient means of converting chemical energy into electrical power. They are named for their solid-oxide electrolytes, which must conduct $\mathrm{O}$ ions from cathode to anode while remaining electrically insulating. The need for high temperatures to achieve sufficient $\mathrm{O}$ conductivity has, however, hampered their application. Alternative electrolytes that can provide high ionic conductivities at lower temperatures are therefore of great interest. Research in bulk materials has achieved some gains in ionic conductivity, but the greatest enhancement has been observed in thin multilayer oxide systems. In particular, up to eight orders of magnitude has recently been reported for thin films of yttria-stabilized zirconia $\left(\mathrm{Y}_{2} \mathrm{O}_{3}\right)_{\mathrm{x}}\left(\mathrm{ZrO}_{2}\right)_{1-\mathrm{x}}$ (YSZ) sandwiched between $\mathrm{SrTiO}_{3}$ (STO) [1].

Aberration correction has produced extraordinary advances in the power of scanning transmission electron microscopy (STEM) to probe the structure and chemistry of materials. It is now possible to chemically map materials with electron energy loss spectroscopy (EELS) on the atomic scale. The structural information gained from aberration corrected STEM is particularly useful when combined with theoretical simulations such as those possible with density functional theory (DFT). DFT can predict both structures and physical properties. We have used the combination of aberration corrected STEM and DFT to elucidate the origin of the colossal ionic conductivity observed in YSZ-STO multilayer systems. Highangle annular dark field (ADF) images of the multilayers with the highest conductivities have displayed laterally continuous YSZ thin films which are strained a large 7\% to match the STO lattice [1]. An example of YSZ strained to match the STO lattice is shown in Fig. 1a. An intensity profile taken along the $\langle 110\rangle$ STO direction across the coherent STO-YSZ-STO layers is shown in the inset. Only small changes in intensity are seen for the atomic columns between the blue arrows, as opposed to the alternating bright and dark intensities of the STO Sr and TiO columns, indicating that the region between the arrows is YSZ. Using DFT to simulate the strained YSZ we find that it has a highly disordered O sublattice either when heated or sandwiched between STO, which has an incompatible O sublattice. Furthermore, by performing finite-temperature quantum molecular dynamics simulations for both strained and unstrained zirconia we find that $\mathrm{O}$ is far more mobile in strained zirconia with the disordered $\mathrm{O}$ sublattice. Strain and O-sublattice incompatibility are therefore responsible for the observed colossal ionic conductivity [2].

Strain and disorder also occur in and around dislocation cores, suggesting they could be used an alternative means of achieving enhanced $\mathrm{O}$ conductivities. Fig. 2 shows dislocation cores formed between islands of YSZ within STO. Areas of YSZ expansion and contraction appear along the interface. The green lines follow the YSZ unit cells from the middle of the island to the interface where the unit cells are expanded. The lattice spacing around the dislocation cores is up to $10 \pm 2 \%$ 
larger than in the center of the island. This strain is even greater than the $7 \%$ of YSZ coherently layered with STO, suggesting it is likely to have a high O conductivity. Spectrum images show the transition to YSZ from STO to be relatively abrupt. We will report ionic conductivity measurements correlated with STEM images of strain from thin films and other specimens with dislocation cores.

\section{References}

[1] J. Garcia-Barriocanal et al., Science 321, 676 (2008).

[2] T.J. Pennycook et al., Phys. Rev. Lett., in press (2010).

[3] Research supported by the DOE Division of Materials Science and Engineering, DOE grant DE-FG02-09ER46554, the McMinn Endowment, NERSC, the Spanish Ministry for Science and Innovation, and the Madrid Regional Government.
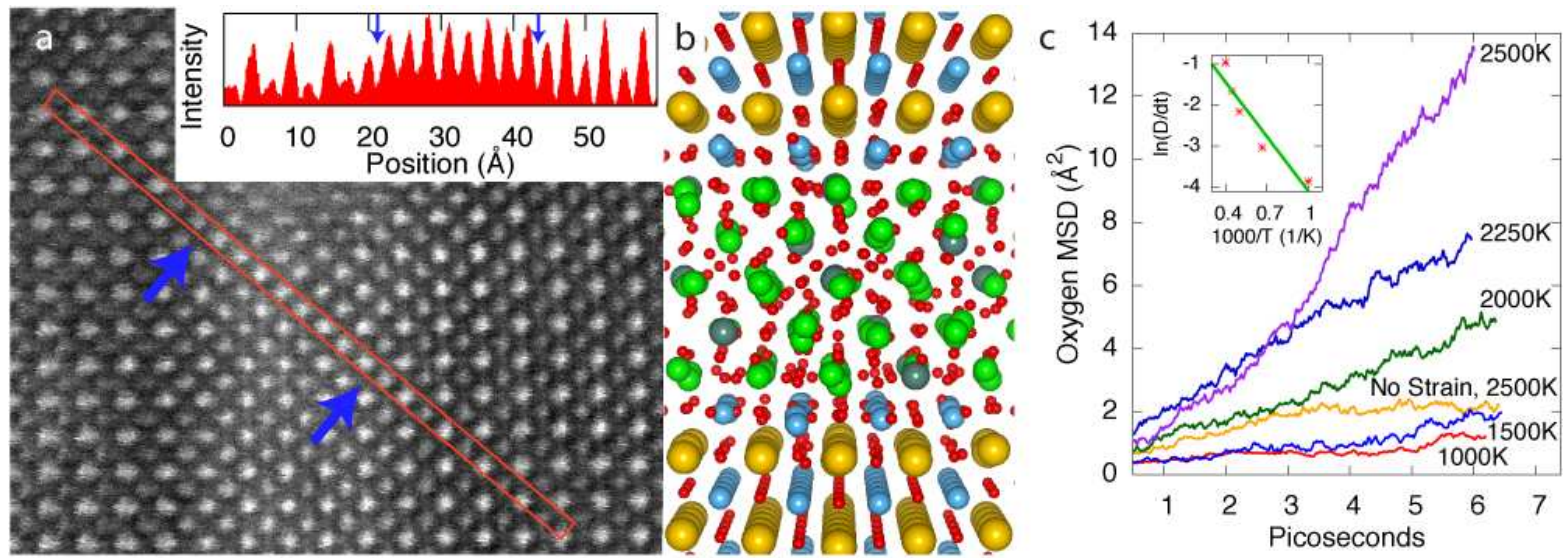

FIG. 1. (a) High resolution ADF image of a YSZ layer coherently strained to match the STO around it. The inset shows the intensity (arbitrary units) as a function of position along the red rectangle in the image. The blue arrows mark the edges of the YSZ layer. (b) Structure resulting from DFT simulation of a thin YSZ layer sandwiched between STO. Sr atoms are shown in yellow, Ti in blue, $\mathrm{Zr}$ in green, $\mathrm{Y}$ in gray, and $\mathrm{O}$ in red. (c) Mean squared displacements of $\mathrm{O}$ ions in strained zirconia at various temperatures compared to that of the unstrained material at $2500 \mathrm{~K}$. Reproduced from [2].

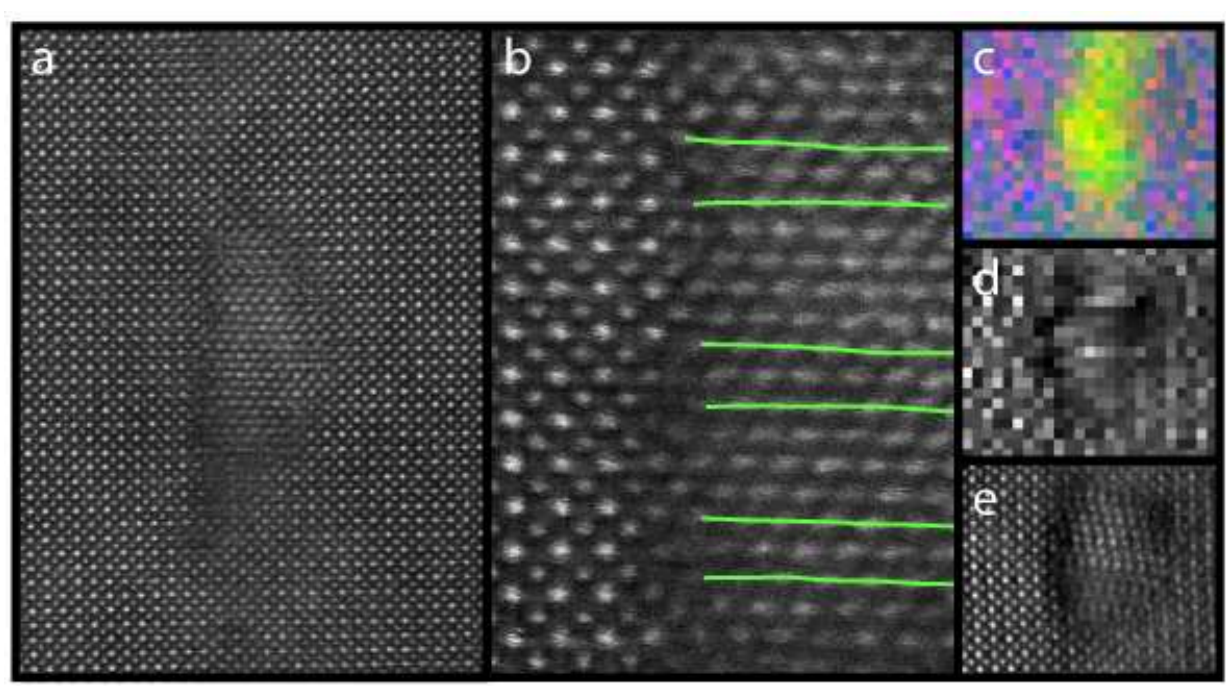

FIG. 2. (a) High resolution ADF image of a YSZ island contained in STO. (b) Closer view of the dislocation cores along the left side of the island. (c) Spectrum image of the YSZ island imaged simultaneously in (d) and at high resolution in (e). The integrated intensities of the $\mathrm{O}, \mathrm{Zr}$, and Ti EELS edges compose the red green and blue channels of the spectrum image. 